\title{
NONSOLVABLE IC GROUPS
}

\section{S. BAUMAN}

Introduction. In this paper we will consider the structure of a nonsolvable group satisfying the condition which I will denote by IC.

Definition. A finite group $G$ is said to be an IC group if for any two nonincident subgroups $H$ and $K, H \cap K$ has only cyclic Sylow groups.

REMARK. Two subgroups $H$ and $K$ are nonincident if $H \nsubseteq K$ and $K \nsubseteq H$.

It follows easily that both subgroups and factor groups of IC groups are themselves IC groups. The result we will prove is the following.

TheOREM. If $G$ is a nonsolvable IC group then $G$ is isomorphic to one of the following:

(i) $\mathrm{SL}(2,4)$,

(ii) $\mathrm{SL}(2,8)$,

(iii) $\operatorname{SL}(2,5)$.

REMARK. In this context a $p$-group will be considered a Sylow group of itself. Thus for a $p$-group an IC group has $H \cap K$ cyclic for all nonincident subgroups $H$ and $K$.

1. We want to consider the structure of a non-abelian IC 2-group. Let $S$ be a 2-group which is an IC group. Suppose $S$ is non-abelian. $S$ contains at least two maximal subgroups $M_{1}$ and $M_{2}$. Since $S$ is IC, $M_{1} \cap M_{2}$ is a cyclic group. We will show that $M_{1} \cap M_{2}$ is the Frattini subgroup of $S$. Suppose this is not the case. Then $S / \Phi(S)$ has order $\geqq 8$. $\Phi(S)$ being the intersection of maximal groups is cyclic and therefore contains a subgroup $R$ of index 2 which is normal in $S$. $S / R$ is not elementary abelian and is of order $\geqq 16$. Consider any subgroup $F / R$ of order 4 in $S / R$. Denote by "-" subgroups of $S$ modulo $R$. If $[\Phi(S)] \cap \bar{F}=\overline{1}$ then $[F \Phi(S)]$ is elementary abelian of order 8 when $\bar{F}$ is noncyclic. We can find an $\bar{M} \subseteq \bar{S}$ such that $(\bar{M}:[F \Phi(S)])=2$. Since $\bar{M}$ has more than one maximal group, we get a contradiction to $\bar{M}$ being an IC group by intersecting $[F \Phi(S)]$ with any other maximal subgroup of $\bar{M}$. If $\bar{F}$ is noncyclic and $[\Phi(S)] \subset \bar{F}$ we have that $F$ is noncyclic containing $\Phi(S)$. Since $(F: \Phi(S))=2$ and $(S: \Phi(S)) \geqq 8$ we get that $F$ is contained in at least two maximal subgroups of $S$. This again contradicts $S$ being IC. Therefore $S / R$ has only cyclic 4 -groups. By a standard result $S / R$ is

Received by the editors April 26, 1963. 
cyclic or quaternion. Since $(S / R:\{1\}) \geqq 16$ and $S / R$ is not cyclic we have a contradiction. Thus $(S / \Phi(S):\{1\})=4$ and $M_{1} \cap M_{2}$ $=\Phi(S)$. Since every maximal subgroup of $S$ must contain $\Phi(S)$ we have that $S$ contains only three maximal subgroups. Denote them by $M_{1}, M_{2}, M_{3}$. We put these conclusions in the form of a lemma.

LemMa 1. Let $S$ be a non-abelian 2-group which is an IC group. Then $S$ is a 2-generator group with cyclic Frattini subgroup.

LEMMA 2. If $G$ is an IC group then $G$ is p-normal for each $p \mid(G: 1)$.

Proof. Let $P$ be a $p$-Sylow group of $G$ for any prime $p \mid(G: 1)$. If $P$ is abelian we are done. Thus assume $P$ non-abelian. We must have that $Z(P)$ is cyclic. If not, then since $P$ is an IC group we have that $P / Z(P)$ is cyclic. From this and a theorem of Zassenhaus we conclude that $P$ would be abelian. This contradicts our assumption. Let $T$ be another $p$-Sylow group of $G$ with $T \neq P$. We will assume $Z(P) \subset T$ and will try to show that $Z(P)=Z(T)$. Since $T$ is nilpotent we have $Z(P) \subset N_{T}(Z(P)) \subseteq T .{ }^{1}$ Since every subgroup of a cyclic group is characteristic we have that either $N_{T}(Z(P))=T$ or $N_{T}(Z(P))$ is noncyclic. If $N_{T}(Z(P))=T$ we have that $Z(P) \triangleleft T$ and as is well known this yields $Z(P)=Z(T)$. If $N_{T}(Z(P))$ is noncyclic and not equal to $T$, then since $N_{T}(Z(P))=T \cap N_{G}(Z(P))$ is noncyclic $T \subseteq N_{G}(Z(P))$ or $N_{G}(Z(P)) \subseteq T$. If the first holds then as before we conclude that $Z(P)=Z(T)$. The second alternative cannot hold since $P \subseteq N_{G}(Z(P))$ and $P \neq T$. Q.E.D.

Lemma 3. If $G$ is an abelian IC group then either $G$ is cyclic or a $p$ group of type $\left(p, p^{n}\right)$ or $(p, p, p)$.

Proof. This follows from the fundamental theorem of abelian groups and the property of being an IC group.

TheOREM 1. Let $G$ be an IC group. One of the following holds:

(1) a 2-Sylow group of $G$ is abelian,

(2) a 2-Sylow group of $G$ is quaternion,

(3) $G$ possesses a normal 2-complement.

Proof. The proof will be by induction on the order of $G$. Assume that the order $G$ is even and the 2-Sylow group is not abelian or quaternion. As is well known then $S$ must contain a noncyclic maximal subgroup. Denote it by $M_{1}$. If $Z(S)$ is the center of $S$, we are done by 2-normality, Grün's theorem and induction when $N_{G} Z(S) \neq G$. There-

\footnotetext{
${ }^{1} \subset$ means strict inclusion.
} 
fore we may assume that $Z(S) \triangleleft G$. Consider $\bar{G}=G / Z(S)$. If $\bar{S}$ is quaternion of order 8 then all the maximal subgroups $\bar{M}_{1}, \bar{M}_{2}$ and $\bar{M}_{3}$ are cyclic. By a theorem of Zassenhaus we may assert that $M_{1}, M_{2}$ and $M_{3}$ are abelian groups. But then $\Phi(S)$ is the intersection of two abelian maximal groups and is thus in the center of $S$. This contradicts the fact that $S / Z(S)$ has order 8 . If $S / Z(S)$ is not abelian we may apply induction to $\bar{G}$ and assert that $\bar{G}$ has a normal 2-complement $\bar{C}$. Since $Z(S)$ is a cyclic 2 -Sylow subgroup of $C$ we get a normal 2-complement in $C$ which serves as a normal 2-complement in $G$. Thus we consider the case where $S / Z(S)$ is abelian. If $N_{G}(S)=S$, then by passing to $G / Z(S)$ and applying a theorem of Burnside we as above get a normal 2-complement in $G / Z(S)$ and hence in $G$. Thus we may assume $N_{G}(S) \supset S .{ }^{2}$ Since $M_{1}$ is not cyclic and since $G$ is an IC group it is not hard to see that $N_{G}\left(M_{1}\right)=S$. Since $N_{G}(S)$ is bigger than $S$ we also have that $M_{1} \cong M_{2} \cong M_{3}$. By a similar argument $M_{1} / Z(S)$ $\cong M_{2} / Z(S) \cong M_{3} / Z(S)$. Since all the maximal groups of abelian $S / Z(S)$ are isomorphic, we must have that $S / Z(S)$ is elementary abelian of order 4 or order 8 . If $S / Z(S)$ is of order 8 we get a contradiction to Lemma 1 . Thus $S / Z(S)$ is a Klein group. As before we conclude that $M_{1}, M_{2}, M_{3}$ are abelian. Since $S$ is not quaternion and $Z(S)$ is cyclic there exist noncentral involutions. Therefore $M_{i}=Z(S) \times \epsilon_{i}$, where $\epsilon_{i}$ are noncentral involutions. There exists a subgroup $R$ such that $(Z(S): R)=2$ and $R$ is normal in $G$. It is not hard to see that $S / R$ is elementary abelian of order 8 . This contradicts Lemma 1 . Thus the theorem is proved. Q.E.D.

We would now like to study the nonsolvable IC groups. By Theorem 1 and the recent result of Feit and Thompson [2] as to the solvability of groups of odd order we see that the two Sylow groups are either abelian or quaternion. By Lemma 3 and a well-known result on cyclic 2-Sylow groups we may assert that the 2-Sylow group is either abelian of type $\left(2,2^{n}\right)$ or elementary abelian of order 8 or quaternion. We will consider each case.

THEOREM $2 .^{3}$ If $G$ is a nonsolvable IC group with an abelian 2-Sylow group then $G$ is isomorphic to $\mathrm{SL}\left(2,2^{2}\right)$ or $\mathrm{SL}\left(2,2^{3}\right)$.

Proof. Consider $\bar{G}=G / G^{\prime}$. Let $G_{0}$ be the inverse image of the 2Sylow complement of $\bar{G}$ in $G$. Let $S_{0}$ be a 2 -Sylow group of $G_{0}$. Since $G / G_{0}$ is an abelian 2-group we must have that $G_{0}$ is a nonsolvable IC group. Suppose $G_{0} \neq G$. Then there is a 2-Sylow group $S$ of $G$ such

2D means strict inclusion.

${ }^{3}$ The author is indebted to the referee for simplifying his proof of Theorem 2 . 
that $S_{0} \subset S$ and $S \nsubseteq G_{0}$. Thus $S_{0} \subseteq S \cap G_{0}$. This contradicts the nonsolvability of $G_{0}$. We therefore have $G=G_{0}$.

Let $S$ be a 2-Sylow group of $G$. Let $x \neq 1$ be any element of $S$. Since $S$ is abelian we have that $S \subseteq C_{G}(X) \cap N_{G}(S)$. The IC property forces $C_{G}(x) \subseteq N_{G}(S)$ or $N_{G}(S) \subseteq C_{G}(x)$. If the latter case occurs, we must have that $S$ intersection the center of $N_{G}(S)$ is not the identity. But by a theorem of Zassenhaus [4, p. 173], the transfer of $G$ into $S$ maps $G$ onto this intersection. This contradicts the fact that $G=G_{0}$. Therefore for all $x \in S, x \neq 1$, we have $C_{G}(x) \subset N_{G}(S)$. Since a group of type $\left\{2,2^{n}\right\}$ with $n>1$ contains a unique noncyclic maximal subgroup and $N_{G}(S) \supset S$ we must have that $S$ is elementary of order 4 or 8 . Again by IC we must have that either $C_{G}(S) \subset C_{G}(x)$ or $C_{G}(x) \subseteq C_{G}(S)$. Suppose the first case occurs. If $|S|=4$, since $\left(N_{G}(S): C_{G}(S)\right)=3$, we get that $C_{G}(x)=N_{G}(S)$. An application of Maschke's theorem on complete reducibility yields $N_{G}(S)=C_{G}(S)$. This contradicts the nonsolvability of $G$. If $|S|=8$ we get that $C_{G}(x)=\mathrm{SL}$, where $|L| \neq 1$. By Maschke's theorem we get that $L$ normalizes a Klein group $V$. Thus $L V \cap S$ contradicts the IC property of $G$. Therefore we must have for all $x \neq 1, x \in S$, that $C_{G}(x) \subseteq C_{G}(S)$. But nonsolvable groups satisfying this property have been characterized by Feit in [3]. Such a group is $\operatorname{SL}\left(2,2^{n}\right)$ for some value of $n$. Since $|S|$ is 4 or 8 we get the result of the theorem. A close inspection verifies that $\operatorname{SL}\left(2,2^{2}\right)$ and $\operatorname{SL}\left(2,2^{3}\right)$ are indeed IC groups.

THEOREM 3. If $G$ is a nonsolvable IC group with a quaternion 2Sylow group then $G \cong \mathrm{SL}(2,5)$.

Proof. By a theorem of Brauer [1], a group $G$ with a quaternion 2-Sylow group contains a normal subgroup $N$ of odd order such that $G / N$ contains a unique involution. Let $T / N$ be the unique and therefore normal subgroup of order 2 in $G / N$. Since $T$ is solvable we must have that $G / T$ is a nonsolvable IC group containing the Klein group as 2-Sylow group. Thus by Theorem $2 G / T$ must be isomorphic to $\mathrm{SL}(2,4)$. Since $G$ is an IC group $T$ has all cyclic Sylow groups. In particular $T$ has a normal 2-complement $R$. If $V$ is a noncyclic 4group of $G$ and $S$ a 2-Sylow group containing $V$ then $R V \cap S$ contradicts the IC property of $G$. Thus we must have that $|R|=1$ and $|T|=2$. By a result of Schur we get that either $G$ is isomorphic to $\mathrm{SL}(2,5)$ or $A_{5} \times T$. The second case is not possible by the structure of the 2-Sylow group. Thus $G$ is isomorphic to $\operatorname{SL}(2,5)$. A check of the subgroup structure of this group verifies that $\operatorname{SL}(2,5)$ is an $\operatorname{IC}$ group.

Putting these results into one theorem we state: 
TheOREM 4. If $G$ is a nonsolvable IC group then $G$ is isomorphic to one of the following:

(i) $\mathrm{SL}\left(2,2^{2}\right)$,

(ii) $\operatorname{SL}\left(2,2^{3}\right)$,

(iii) $\operatorname{SL}(2,5)$.

\section{REFERENCES}

1. R. Brauer and M. Suzuki, On finite groups of even order whose 2-Sylow group is a quaternion group, Proc. Nat. Acad. Sci. U.S.A. 45 (1959), 1757-1759.

2. W. Feit and J. H. Thompson, Solvability of groups of odd order, Pacific J. Math. 13 (1963), 775-1029.

3. W. Feit, $A$ characterization of the simple groups $\mathrm{SL}\left(2,2^{a}\right)$, Amer. J. Math. 82 (1960), 281-300.

4. H. Zassenhaus, The theory of groups, 2nd ed., Chelsea, New York, 1958.

PRINCETON UNIVERSITY 